\title{
Heat transfer analysis of nanofluid flow through backward facing step
}

\author{
Ahlem Boudiaf ${ }^{1,{ }^{*}}$, Fetta Danane ${ }^{1}$, Youb Khaled B enkahla ${ }^{1}$, Walid Berabou ${ }^{1}$, Mahdi Benzema ${ }^{1}$, and Seif-Eddine Ouyahia ${ }^{1}{ }^{2}$ \\ ${ }^{1}$ University of Science and Technology Houari B oumediene, 16111 A lgiers, A LGERIA \\ ${ }^{2}$ Central Directorate Research and Development, Sonatrach, 35000 B oumerdes, ALGERIA
}

\begin{abstract}
This paper presents the numerical predictions of hydrodynamic and thermal characteristics of nanofluid flow through backward facing step. The governing equations are solved through the finite volume method, as described by Patankar, by taking into account the associated boundary conditions. Empirical relations were used to give the effective dynamic viscosity and the thermal conductivity of the nanofluid. Effects of different key parameters such as Reynolds number, nanoparticle solid volume fraction and nanoparticle solid diameter on the heat transfer and fluid flow are investigated. The results are discussed in terms of the average N usselt number and streamlines.
\end{abstract}

\section{Introduction}

Heat transfer enhancement using nanoparticles have gained the attention of several researchers. The mixture of these nanoparticles with a base fluid gives birth to nanofluid which responded the need to improve the thermal diffusivity of the fluid using metals and oxides with better thermal conductivity.

Many experimental and numerical studies have considered nanofluid in various kinds of geometries

Benzema et al. [1] reported a numerical investigation of mixed convection in an irregular ventilated cavity filled with $\mathrm{Cu}$-Water nanofluid. They concluded that the heat transfer rate is enhanced by increasing the Ri number, Re number and nanoparticles volume fraction but decreases with the nanoparticles diameters increase.

Considering a backward facing step, Abu-Nada [2] carried out a numerical analysis of forced convection of nanofluid using various types of nanoparticles. He noticed that the Re number has no effect on the heat transfer rate inside the recirculation zone and that nanoparticles with low thermal conductivity present higher value of the Nusselt number. The same study has been undertaking by Al-aswadi et al. [3], they examined the Re number and nanoparticles types effects on the static pressure and the skin friction coefficient.

A mixed convection of nanofluid flow over a backward facing step with slotted baffle has been investigated by Heshmati et al. [4]. They noticed that the inclined slotted baffle is the most advantageous geometry with an appropriate Nusselt number and minimal pressure drop. An experimental study has been made by Abbasian [5] to examine the heat transfer and fluid flow characteristics of $\mathrm{TiO} 2-$ Water nanofluid. They found that the best diameter of $\mathrm{TiO} 2$ nanoparticles is $20 \mathrm{~nm}$ that presents the highest heat transfer rate. Many other works have focused on the heat transfer characteristics of nanofluid [6]-[8].

The aim of this study is to examine the heat transfer characteristics of nanofluid flow over backward facing step when a portion of the bottom wall is exposed to uniform heat flux.

\section{Analysis and modelling}

The configuration of problem under investigation "Figure 1" includes suspension of $\mathrm{TiO} 2$ nanoparticles and Newtonian base fluid through a backward facing step. A portion of the lower wall is maintained at uniform heat flux whereas the remaining walls of the channel are kept insulated. Besides, the thermophysical properties of the nanofluid (given in Table1) are assumed to be constant except the thermal conductivity which will vary with the temperature using Corcion's formulation [9].

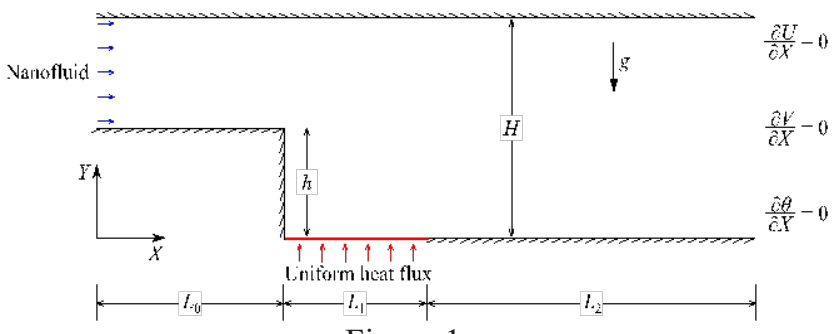

Figure 1

Fig. 1. Schematic of the physical domain.

Under the assumptions cited above, the corresponding non-dimensional governing equations are written as follows:

* Corresponding author: ahboudiaf@ usthb.dz 


$$
\begin{gathered}
\frac{\partial u}{\partial x}+\frac{\partial v}{\partial y}=0 \\
\rho_{n f}\left(u \frac{\partial u}{\partial x}+v \frac{\partial u}{\partial y}\right)=-\frac{\partial p}{\partial x}+\left[2 \frac{\partial}{\partial x}\left(\mu_{n f} \frac{\partial u}{\partial x}\right)+\right. \\
\left.\frac{\partial}{\partial y}\left(\mu_{n f}\left(\frac{\partial u}{\partial y}+\frac{\partial v}{\partial x}\right)\right)\right] \\
\rho_{n f}\left(u \frac{\partial v}{\partial x}+v \frac{\partial v}{\partial y}\right)=-\frac{\partial p}{\partial y}+\left[2 \frac{\partial}{\partial y}\left(\mu_{n f} \frac{\partial v}{\partial y}\right)+\right. \\
\left.\frac{\partial}{\partial x}\left(\mu_{n f}\left(\frac{\partial u}{\partial y}+\frac{\partial v}{\partial x}\right)\right)\right] \\
\left(\rho c_{p}\right)_{n f}\left(u \frac{\partial T}{\partial x}+v \frac{\partial T}{\partial y}\right)=k_{n f}\left(\frac{\partial^{2} T}{\partial x^{2}}+\frac{\partial^{2} T}{\partial y^{2}}\right)
\end{gathered}
$$

The effective viscosity of nanofluid was be modeled by the experimental correlation given by Corcione[9].

$$
\mu_{n f}=\frac{\mu_{f}}{1-34.87\left(\frac{d_{p}}{d_{f}}\right)^{-0.3} \phi^{1.03}}
$$

$\mu_{f}$ is the apparent viscosity of the base fluid.

The same thing for the effective thermal conductivity of the nanofluid, an experimental correlation proposed by Corcione was be used.

$$
k_{n f}=k_{f}\left[1+4.4 \operatorname{Re}_{B}^{0.4} \operatorname{Pr}^{0.06}\left(\frac{T}{T_{f r}}\right)^{10}\left(\frac{k_{p}}{k_{f}}\right)^{0.03} \phi^{0.66}\right]
$$

where $R e_{b}$ is the nanoparticle Reynolds number defined as:

$$
\operatorname{Re}_{B}=\frac{\rho_{f} U_{B} d_{p}}{\mu_{f}}
$$

$\rho_{f}$ and $\mu_{f}$ are the mass density and the dynamic viscosity of the base fluid, respectively, while $U_{B}$ is the mean Brownian velocity.

$$
U_{B}=\frac{2 k_{b} T}{\pi \mu_{f} d_{p}^{2}}
$$

The other parameters are given as follows:

$$
\begin{gathered}
\rho_{n f}=\rho_{s} \phi+\rho_{f}(1-\phi) \\
\left(\rho c_{p}\right)_{n f}=\left(\rho c_{p}\right)_{s} \varphi+\left(\rho c_{p}\right)_{f}(1-\phi) \\
(\rho \beta)_{n f}=(\rho \beta)_{s} \phi+(\rho \beta)_{f}(1-\phi)
\end{gathered}
$$

Table 3. Thermophysical properties of base fluid and $\mathrm{TiO}_{2}$.

\begin{tabular}{ccccc}
\hline & $\begin{array}{c}\rho \\
\left(\mathrm{kg} / \mathrm{m}^{3}\right)\end{array}$ & $\begin{array}{c}k \\
(\mathrm{~W} / \mathrm{m} \mathrm{K})\end{array}$ & $\begin{array}{c}c_{p} \\
(\mathrm{~J} / \mathrm{kg} \mathrm{K})\end{array}$ & $\begin{array}{c}\beta \times 10^{5} \\
\left(\mathrm{~K}^{-1}\right)\end{array}$ \\
\hline base fluid & 1002 & 0.6 & 4500 & 21 \\
$\mathrm{TiO}_{2}$ & 4250 & 8.9538 & 686.2 & 0.9 \\
\hline
\end{tabular}

$$
\begin{aligned}
& \frac{\partial U}{\partial X}+\frac{\partial V}{\partial Y}=O \\
& U \frac{\partial U}{\partial X}+V \frac{\partial U}{\partial Y}=-\frac{\partial P}{\partial X}+\frac{\rho_{f}}{\rho_{n f}} \frac{1}{R e}\left[2 \frac{\partial}{\partial X}\left(\mu_{n f} \frac{\partial U}{\partial X}\right)+\right. \\
& \left.\frac{\partial}{\partial Y}\left(\mu_{n f}\left(\frac{\partial U}{\partial Y}+\frac{\partial V}{\partial X}\right)\right)\right] \\
& U \frac{\partial V}{\partial X}+V \frac{\partial V}{\partial Y}=-\frac{\partial P}{\partial Y}+\frac{\rho_{f}}{\rho_{n f}} \frac{1}{R e}\left[2 \frac{\partial}{\partial Y}\left(\mu_{n f} \frac{\partial V}{\partial Y}\right)+\right. \\
& \left.\frac{\partial}{\partial X}\left(\mu_{n f}\left(\frac{\partial U}{\partial Y}+\frac{\partial V}{\partial X}\right)\right)\right] \\
& U \frac{\partial \theta}{\partial X}+V \frac{\partial \theta}{\partial Y}=\frac{\left(\rho c_{p}\right)_{f}}{\left(\rho c_{p}\right)_{n f}} \frac{1}{\operatorname{Re} P r}\left[\frac{\partial \theta}{\partial X}\left(\frac{k_{n f}}{k_{f}} \frac{\partial \theta}{\partial X}\right)+\right. \\
& \left.\frac{\partial \theta}{\partial Y}\left(\frac{k_{n f}}{k_{f}} \frac{\partial \theta}{\partial Y}\right)\right]
\end{aligned}
$$

The local Nusselt number along the lower wall is derived as:

$$
N u=-\left.\frac{k_{n f}}{k_{f}} \frac{\partial \theta}{\partial Y}\right|_{Y=0}
$$

\section{Boundary conditions:}

At the inlet $\mathrm{X}=0$ and $0<\mathrm{Y}<1$

$U=6 \frac{\left[Y^{2}-(h+1) Y+h\right]}{4 h-(h+1)^{2}} ; \quad V=0 ; \quad \theta=0$

At the outlet $X=L$ and $0<Y<1$

$\frac{\partial U}{\partial X}=0 ; \quad \frac{\partial V}{\partial X}=0 ; \quad \frac{\partial \theta}{\partial X}=0$

The lower wall $Y=0,0<X<L_{0}$

$U=0 ; \quad V=0 ; \quad \frac{\partial \theta}{\partial Y}=0$

The lower wall $Y=0, L_{0}<X<L_{1}$

$U=0 ; \quad V=0 ; \quad \frac{\partial \theta}{\partial Y}=-1$ 
The lower wall $Y=0, L_{1}<X<L_{2}$

$$
U=0 ; \quad V=0 ; \quad \frac{\partial \theta}{\partial Y}=0
$$

Along the upper wall $Y=1,0<X<L$

$$
U=0 ; \quad V=0 ; \quad \frac{\partial \theta}{\partial X}=0
$$

\section{Numerical modelling}

The finite volume method was used to discretize the governing system of equations (12)-(15) whereas the SIMPLE algorithm assisted to handle the pressurevelocity coupling. The discretized equations are solved by the line by line tri diagonal matrix algorithm (TDMA). A non-uniform grid size with finer grids at the walls was adopted.

Convergence is declared when the maximum relative change between two consecutive iteration level fall below $10^{-6}$, for $\mathrm{U}, \mathrm{V}, \mathrm{P}$ and $\theta$. In order to validate our computational code accuracy, average Nusselt number was compared with the study of Santra et al.[10] for different volume fraction and Reynold number. As may be seen on "Figure 2" the results are in excellent agreement with a maximum discrepancy of about $2.5 \%$.

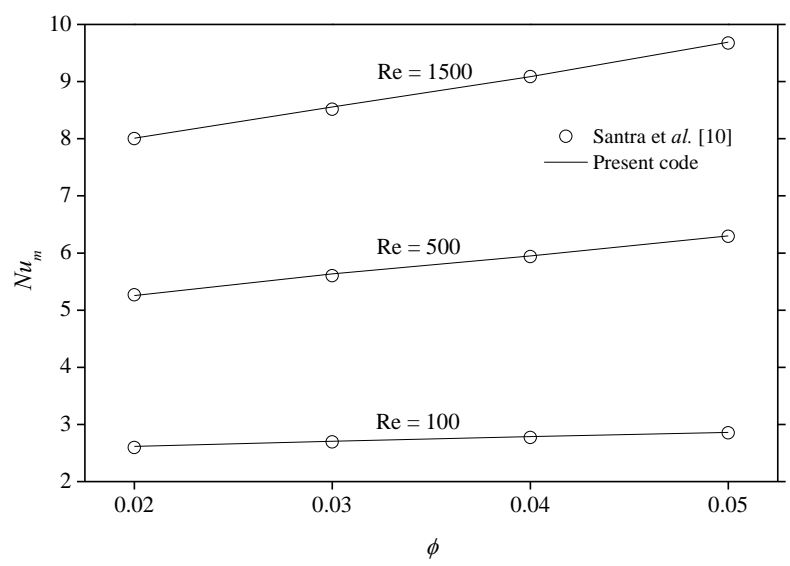

Fig. 2. Comparison of average N usselt number obtained by Santra et al. [10] and those of the present code.
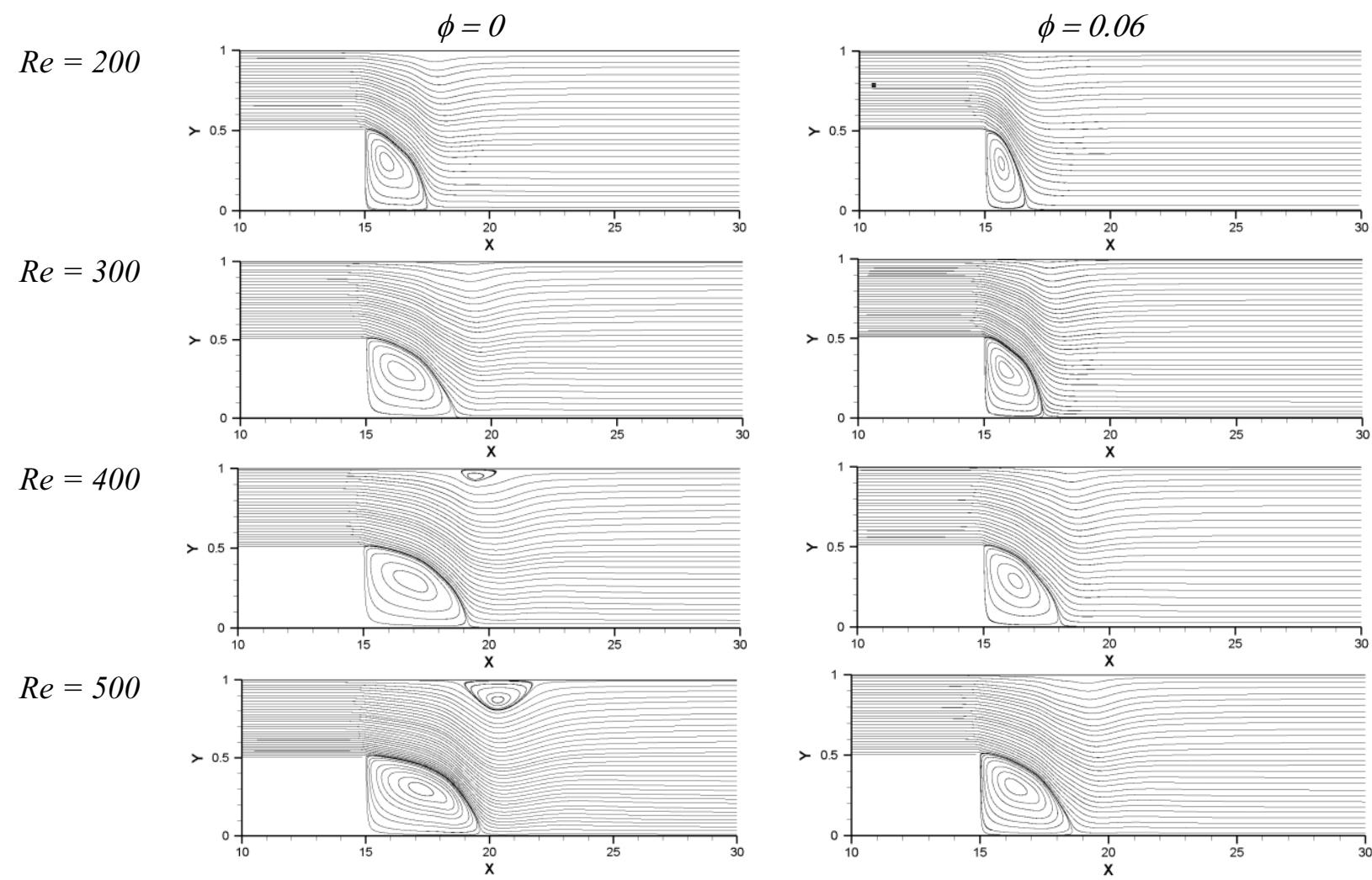

$R e=600$
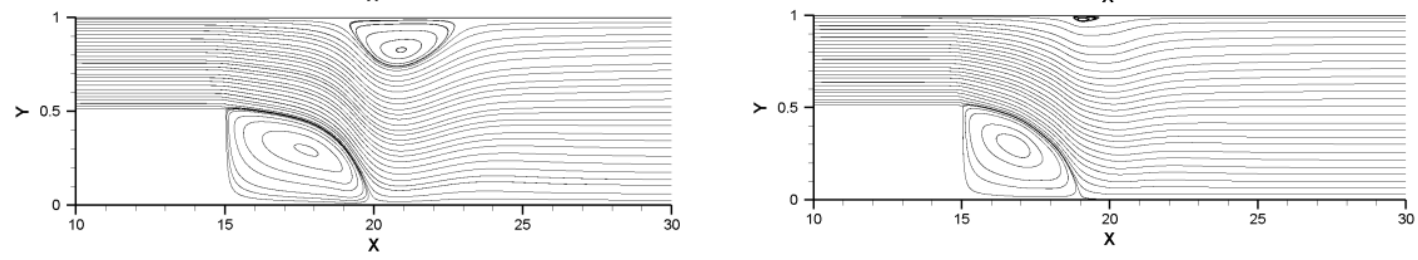

Fig. 3. Streamlines for different Re number, $\phi=0$ and $\phi=0.06$ 


\section{Results and discussion}

The heat transfer and flow characteristics of nanofluid over backward facing step have been investigated in this study. All computations are carried out for $\operatorname{Pr}=10$.

Figure 3 shows the effect of the Re number on the streamlines for $\phi=0$ and $\phi=0.06$. For all studied Re values and $\phi=0$, it is observed the formation of recirculation zone behind the step. The size of this zone increases with the increase of the Re number value. Also it is observed the formation of new recirculation zone near the upper wall for $R e=400$ and which increases in size with the increase in the Re number. We can explain these results by the fact that increasing the Re number leads to an increase in the fluid velocity which enhances to increase the recirculation zone size.

The same observations are made for $\phi=0.06$ where a recirculation zone is created behind the step and gets larger in size with increasing the Re number. While the recirculation zone near the upper wall is observed for $R e=600$ here.

Now comparing the two nanoparticles volume fraction ( $\phi=0$ and $\phi=0.06)$ it is noticed that the recirculation zone size is greater for $\phi=0$ than $\phi=0.06$. This is due to the presence of nanoparticles which decrease the velocity and consequently the recirculation zone size.

Considering the heat transfer, the figure 4 presents the average Nusselt number for different Re number and that for $\phi=0$ and $\phi=0.06$. It is observed that for the pure fluid and the nanofluid, the Nusselt number increases with increasing the Re number. By increasing the Re number, the velocity of the fluid increases and so the temperature gradient between the fluid and the heated wall. It is also observed that the nanofluid $(\phi=0.06)$ presents the highest values of the Nusselt number compared to the pure fluid $(\phi=0.0)$. This is because the nanoparticles lead to increase the thermal conductivity of the fluid.

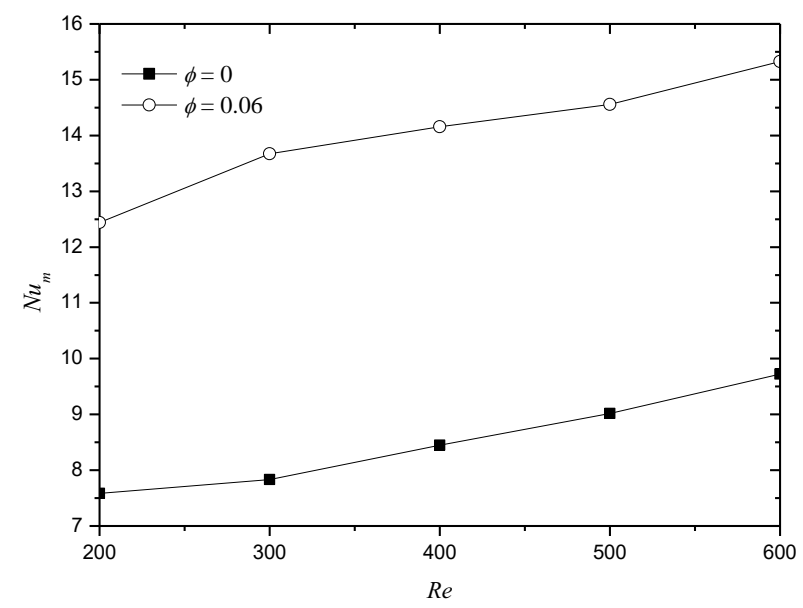

Fig. 4. A verage Nusselt number for different $R e$ number $\phi=0.0$ and $\phi=0.06$.
It is noticed that when the Re number increases, the presence of nanoparticles enhances more the heat transfer rate compared to the base fluid (without nanoparticles).

The figure 5 presents the average Nusselt number for different nanoparticles volume fraction for $R e=400$ and $R e=300$.

It is observed that increasing the nanoparticles volume fraction leads to an increase in the average Nusselt number, this can be explain by the fact that increase the nanoparticles volume fraction increases the thermal conductivity of the fluid which enhance the average Nusselt number.

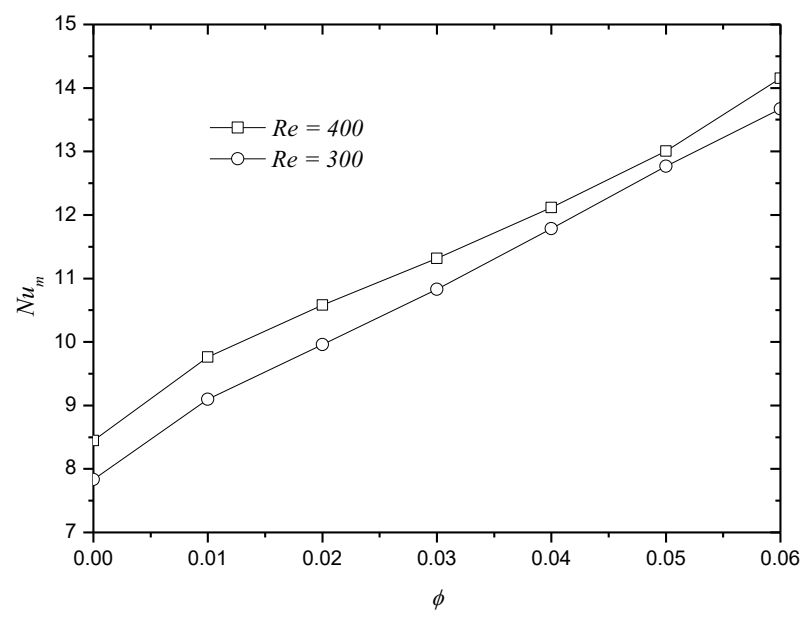

Fig. 5. A verage Nusselt number for different nanoparticles volume fraction $\mathrm{Re}=300$ and $\mathrm{Re}=400$.

Figure 6 illustrates the effect of nanoparticles diameters on the average Nusselt number for two different nanoparticles volume fraction ( $\phi=0$ and $\phi=0.06)$.

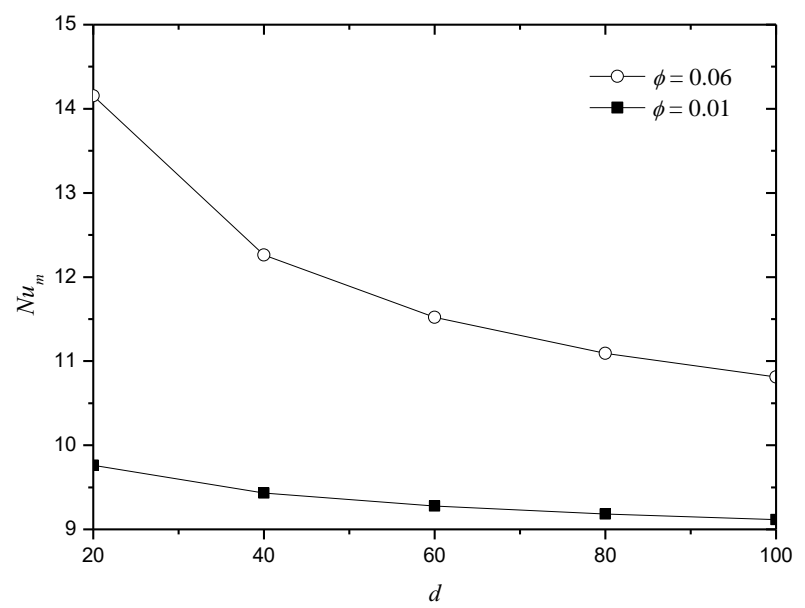

Fig. 6. A verage Nusselt number for different nanoparticles diameters $\phi=0.01$ and $\phi=0.06$.

It is shown that increasing the nanoparticles diameters decreases the average Nusselt number. It is clear that the less nanoparticle diameter $d=20 \mathrm{~nm}$ presents the highest heat transfer rate. It can be explained as for a

\footnotetext{
* Corresponding author: ahboudiaf@ usthb.dz
} 
fixed nanoparticle volume fraction, the nanoparticles with small diameter $(d=20 \mathrm{~nm})$ are more numerous than nanoparticles with high diameter which offers a better mix of the fluid and consequently higher average Nusselt number.

In addition, for $\phi=0.06$ the average Nusselt decrease strongly from diameter to other, for example from $d=20$ $\mathrm{nm}$ to $d=40 \mathrm{~nm}$, while this reduction is not so important for $\phi=0.01$.

It is also observed that for a given nanoparticle diameter, the higher value of the average Nusselt number is observed for the higher nanoparticle volume fraction.

\section{Conclusions}

A numerical study of thermal characteristics of nanofluid through backward facing step has been considered. Wide ranges of Re number, nanoparticles volume fraction and nanoparticles diameters have been examined. The main conclusions are as follow:

- Heat transfer is enhanced by increasing Re number, nanoparticles volume fraction and by decreasing the nanoparticles diameters.

- For the nanofluid $(\phi=0.06)$, the heat transfer enhancement is more important when the Re number augment comparing to the base fluid ( $\phi=0.0)$.

- When the nanoparticles diameter decreases, the heat transfer decreases strongly for $\phi=0.06$ comparing to $\phi=0.01$.

\section{References}

1. M. Benzema, Y. K. Benkahla, N. Labsi, E. Brunier, and S.-E. Ouyahia, Arab. J. Sci. Eng, 1-12 (2017)

2. E. Abu-Nada, Int. J. Heat Fluid Flow 29, 242-249 (2008)

3. A. A. Al-aswadi, H. A. Mohammed, N. H. Shuaib, and A. Campo, Int. Commun. Heat Mass Transf 37, 950-957 (2010)

4. A. Heshmati, H. A. Mohammed, and A. N. Darus, Appl. Math. Comput 240, 368-386 (2014)

5. A. A. Abbasian Arani and J. Amani, Exp. Therm. Fluid Sci 44, 520-533 (2013)

6. R. Saidur, S. N. Kazi, M. S. Hossain, M. M. Rahman, and H. A. Mohammed, Renew. Sustain. Energy Rev 15, 310-323 (2011)

7. B. H. Salman, H. A. Mohammed, K. M. Munisamy, and A. S. Kherbeet, Renew. Sustain. Energy Rev 28, 848-880 (2013)

8. K. Bashirnezhad, M. Ghavami, and A. A. A. A. Alrashed, J. Mol. Liq 244, 309-321 (2017)

9. M. Corcione, Energy Convers. Manag 52, 789-793 (2011)

10. A. K. Santra, S. Sen, and N. Chakraborty, Int. J. Therm. Sci 48, 391-400 (2009) 